\title{
Formation of Ultra Narrow Lamellar Structures in POSS-containing Triblock Terpolymers
}

\author{
Raita Goseki, Takashi Ishizone, Akira Hirao and Teruaki Hayakawa* \\ Department of Organic and Polymeric Materials \\ Graduate School of Science and Engineering, Tokyo Institute of Technology, \\ 2-12-1-S8-36 O-okayama, Meguro-ku, Tokyo 152-8552, Japan
}

\begin{abstract}
An ultra narrow line structure was prepared in the lamellar morphology of polyhedral oligomeric silsesquioxane (POSS)-containing triblock terpolymer in the bulk via the self-assembly. The triblock terpolymers, which comprised polystyrene, POSS-containing poly(methacrylate) (PMAPOSS) and poly(methyl methacrylate), were synthesized by anionic polymerization. The self-assembled structures were studied by using a transmission electron microscopy (TEM) and a small-angle X-ray scattering (SAXS). It was found that a narrow PMAPOSS domain was layered in triple-domain lamellar, which caused by the effect of strongly segregation with the middle PMAPOSS block. The total $d$-spacing was $32 \mathrm{~nm}$, and the smallest width of PMAPOSS domain layer was ca. $4 \mathrm{~nm}$.
\end{abstract}

Keyword: triblock terpolymer, polyhedral oligomeric silsesquioxane (POSS), self-assembly

\section{Introduction}

Block copolymer lithography exploits the self-assembled nanostructures formed in block copolymers thin films for producing dense nanopatterns over a large area that may overcome the intrinsic resolution limit of conventional optical lithography [1-3]. For the practical utilization of the block copolymer lithography, the directed self-assembly of block copolymers offers a promising method to form the long range highly ordered nanopatterns such as dots and lines in the thin films based on epitaxial assembly on chemically and/or topographically patterned substrates. The pattern sizes are generally dependent on the domain spacing of the microphase-separated nanostructures $[4,5]$. Therefore, achieving the high resolution patterns is required the use of strongly segregating block copolymers that have a relatively low molecular weight with a high $\chi$ parameter.

Recently, we had reported the development of and self-assembly of a novel series of polyhedral oligomeric silsesquioxane (POSS)-containing polymethacrylate (PMAPOSS)-based diblock copolymers such as polystyrene (PS)-blockPMAPOSS, poly(methyl methacrylate) (PMMA)-block-PMAPOSS for the block copolymer lithography materials [6-8]. They can self-assemble to well-defined features of a $10 \mathrm{~nm}$ scale in the thin films because of the strongly segregating ability [9]. Furthermore, the PMAPOSS domains have significantly higher etch resistance compared with the organic domains to oxygen reactive ion etching.

Line patterning in lithography is one of the most important and useful for a wide variety of devices. Creating the single nanometer scale narrow lines with a high resolution is required in the near future of the block copolymer lithography. The line patterns are generally produced by 
selective etching of the microphase-separated nanostructures with parallel cylinders or oriented lamellar in the thin films. However, the cylindrical structures that comprise the minor domains of the PMAPOSS segments were not observed in the bulk morphologies of PMAPOSS-containing block copolymers. This was in spite of the fact that the structures of the microphase-separated PMAPOSS-containing polymers could be varied from spheres to lamellae by altering the PMAPOSS volume fraction. On the other hands, the lamellar structures were obtained in a wide range of volume fraction. However, we have not successfully obtained the oriented lamellar in the thin films for PMAPOSS-containing diblock copolymers.

In this study, we propose the $\mathrm{ABC}$ triblock copolymer with PMAPOSS as a short middle block (B) in order to build progressively more advanced architecture leading to tailored line patterns based on the oriented lamellar structures. Each A and C block was selected PS and PMMA, respectively, because it is well-known that the PS- $b$-PMMA diblock copolymer can form the oriented lamellar structures in the simple thermal annealing. Therefore, we came up with idea that the ABC triblock copolymers consisting of PS (A), PMAPOSS (B) and PMMA (C), which can basically form the lamellar structures of PS and PMMA, can exploit for creating line patterns by selective etching of PS and PMMA domains in the oriented lamellar structures in the thin films. In this case, we can expect to obtain the narrow lines with a single nanometer width by remaining the strong segregated and the oxidized PMAPOSS domains that exist as an interface layer between the PS and PMMA lamellar structures.

Herein, the procedure for synthesizing a new series of POSS-containing triblock terpolymers, PS- $b$-PMAPOSS- $b$-PMMAs, by sequential anionic polymerization is presented in a preliminary account. The self-assembled structures obtained using the triblock terpolymers microphase separated nanostructures in the bulk was studied by small-angle X-ray scattering (SAXS) and transmission electron microscopy (TEM).

\section{Experimental}

\subsection{Materials}

Sec-Butyl lithium (sec-BuLi) was purchased from Kanto Chemical Co (Tokyo,
Japan). $\quad 3-(3,5,7,9,11,13,15$-Heptaisobutylpentacyclo-[9.5.1. ${ }^{3,9} 1 .^{5,15} 1^{7,13}$ ]octasiloxan-1-y 1)propyl methacrylate (MAPOSS) was purchased from Hybrid Plastics Inc. (USA). The other reagents were purchased from TCI Co. (Tokyo, Japan). Prior to use, tetrahydrofuran (THF) was refluxed over $\mathrm{Na}$ wire for $12 \mathrm{~h}$ and distilled over $\mathrm{LiAlH}_{4}$ under nitrogen. It was finally distilled from its sodium naphthalenide solution on a high vacuum line $\left(10^{-6}\right.$ Torr $)$. Styrene was washed with $10 \% \mathrm{NaOH}$ aq, dried over $\mathrm{MgSO}_{4}$, and distilled over calcium hydride $\left(\mathrm{CaH}_{2}\right)$ under reduced pressures. Prior to use, the monomer was distilled over di- $n$-butylmagnesium $\left(\mathrm{Bu}_{2} \mathrm{Mg}\right)(\sim 5 \mathrm{~mol} \%)$ on the vacuum line into ampules equipped with break seals that were prewashed with potassium naphtalenide in THF. Methyl methacrylate (MMA) was first distilled over $\mathrm{CaH}_{2}$ under reduced pressures and then distilled from their $3 \mathrm{~mol} \%$ $\left(\mathrm{C}_{2} \mathrm{H}_{5}\right)_{3} \mathrm{Al}$ solution on the high vacuum line. 1,1-diphenylethylene (DPE) was distilled over $n$-butyllithium, MAPOSS was purified by recrystallization from methanol and dried on the high vacuum line with diphosphorous pentaoxide for over night. Lithium chloride (LiCl) was baked under vacuum at $180^{\circ} \mathrm{C}$ for $24 \mathrm{~h}$. The other reagents were used as received.

\subsection{Instrumentation and Characterization}

IR spectra were recorded on a JASCO FT/IR-460 Plus spectrometer. ${ }^{1} \mathrm{H},{ }^{13} \mathrm{C}$ and ${ }^{29} \mathrm{Si}$ NMR spectra were recorded on a JEOL JNM-AL 300 spectrometer at $300 \mathrm{MHz}, 75$ $\mathrm{MHz}$ and $59.4 \mathrm{MHz}$, respectively. Molecular weights and molecular weight distributions were measured on an Asahi Techneion AT-2002 equipped with a Viscotek TDA model 302 triple detector array using THF as a carrier solvent at a flow rate of $1.0 \mathrm{~mL} \mathrm{~min}{ }^{-1}$ at $30{ }^{\circ} \mathrm{C}$. Three PS gel column (pore size (bead size)) were used: $650 \AA$ $(5 \mu \mathrm{m}), 200 \AA(5 \mu \mathrm{m}), 75 \AA(5 \mu \mathrm{m})$. The relative molecular weights were determined by SEC with RI detection using standard polystyrene calibration curve. Thermal analyses of the compounds, thermogravimetric analysis (TGA), and differential scanning calorimetry (DSC) were carried out using a Seiko SSC/6000 (TG/DTA 6200 and DSC6200) thermal analyzer with ca. $5 \mathrm{mg}$ of samples at a heating rate of $10{ }^{\circ} \mathrm{C} \mathrm{min}^{-1}$. Nitrogen was 
used as the purge gas at a flow rate of $50 \mathrm{~mL}$ $\min ^{-1}$ for both the TGA and DSC measurements. For TEM analysis, bulk samples were embedded in epoxy resin and cured at $70{ }^{\circ} \mathrm{C}$ for $24 \mathrm{~h}$. The embedded samples were then microtomed using a Diatome diamond knife at room temperature to a preset thickness of $70 \mathrm{~nm}$ using Microtome. The sections were placed on TEM grids and viewed directly using a JEOL JEM-200CX at $100 \mathrm{kV}$. Small angle X-ray scattering (SAXS) measurements on the bulk samples were carried out obtained using a Bruker NanoSTAR $(50 \mathrm{kV} / 100 \mathrm{~mA})$ with a 2D-PSPC detector (camera length $1055 \mathrm{~nm}$ ).

\subsection{Bulk sample Preparation}

The sample of PS- $b$-PMAPOSS- $b$-PMMA for investigating the bulk morphology was prepared by slow evaporation from chloroform solution and subsequently annealed at $180^{\circ} \mathrm{C}$ in vacuum for $24 \mathrm{~h}$.

\subsection{Synthesis of PS- $b$-PMAPOSS- $b$-PMMA by} Living Anionic Polymerization

All of polymerizations were carried out under high vacuum $\left(10^{-6}\right.$ Torr $)$ in sealed glass reactors equipped with break-seals. A small portion was always taken to determine the molecular weight $\left(M_{\mathrm{n}}\right)$ and molecular weight distribution $\left(M_{\mathrm{w}} / M_{\mathrm{n}}\right)$ values prior to the next reaction. The concentrations of monomer and initiator were usually in the ranges $0.4-0.8$ and $0.02-0.11 \mathrm{M}$, respectively. All reactors were prewashed with red-colored 1,1-diphenylhexyllithium (ca. $0.05 \mathrm{M}$ ) in heptane after being sealed off from a vacuum line and used.

Polystyryllithium (PSLi) was prepared by the polymerization of styrene $(19.0 \mathrm{mmol})$ in THF solution $(22.3 \mathrm{~mL})$ initiated with sec-BuLi $(0.096$ mmol) in heptane solution $(1.60 \mathrm{~mL})$ at $-78{ }^{\circ} \mathrm{C}$ for $10 \mathrm{~min}$. Then, a THF solution $(2.01 \mathrm{~mL})$ of DPE $(0.301 \mathrm{mmol})$ was added to the PSLi solution at $-78^{\circ} \mathrm{C}$. After the reaction was allowed to stand for an additional $30 \mathrm{~min}$ at $-78{ }^{\circ} \mathrm{C}, \mathrm{LiCl}(0.359 \mathrm{mmol})$ dissolved in THF $(3.61 \mathrm{~mL})$ was added $-78{ }^{\circ} \mathrm{C}$, followed by addition of a THF solution $(5.7 \mathrm{~mL})$ of MAPOSS $(0.50 \mathrm{mmol})$ at $-78{ }^{\circ} \mathrm{C}$ with vigorous shaking, and the polymerization was allowed to stand for $6 \mathrm{~h}$. After $6 \mathrm{~h}$, a THF $(21.3 \mathrm{~mL})$ solution of MMA (19.1 mmol) was added with vigorous shaking at $-78^{\circ} \mathrm{C}$, and the polymerization allowed to stand for $30 \mathrm{~min}$. The polymer was quenched with degassed methanol and precipitated in methanol. It was reprecipitated twice from THF to methanol, and freeze-dried from its dry benzene solution in vacuo for $24 \mathrm{~h}$. The polymer $(4.03 \mathrm{~g})$ was obtained in $93 \%$ yield and characterized by SEC, ${ }^{1} \mathrm{H},{ }^{13} \mathrm{C}$, and ${ }^{29} \mathrm{Si}$ NMR, and IR spectroscopy. For final triblock terpolymer, SEC gives $M_{\mathrm{n}}$ (SEC) of $44700 \mathrm{~g} \mathrm{~mol}^{-1}$ and $M_{\mathrm{w}} / M_{\mathrm{n}}$ of 1.04. IR (KBr, $\mathrm{cm}^{-1}$ ): 3082, 3060, 3025, 3000, 2951, 2924, 2849, $1731,1601,1493,1452,1366,1270,1231,1167$, $1115,1028 .{ }^{1} \mathrm{H}$ NMR $\left(300 \mathrm{MHz}, \mathrm{CDCl}_{3} \mathrm{ppm}\right): \delta$ 7.06-7.03 (m, aromatic, PS), 6.57-6.36 (m, aromatic, PS), 3.81 (br, $-\mathrm{OCH}_{2}$, PMAPOSS), 3.59 (br, - $\left.\mathrm{OCH}_{3}, \mathrm{PMMA}\right), 2.06-1.72$ (m, isobutyl $-\mathrm{CH}$ (PMAPOSS), main chain $-\mathrm{CH}_{2},-\mathrm{CH}$, PS, PMAPOSS, PMMA), 1.4-1.39 (m, main chain $-\mathrm{CH}_{2}$, PS), 1.01-0.94 (br, isobutyl $-\mathrm{C}\left(\mathrm{CH}_{3}\right)_{2}$, PMAPOSS), 0.84 (m, $\alpha \mathrm{CH}_{3}$, PMMA), 0.60 (br, -SiCH $\mathrm{H}_{2}$, PMAPOSS). ${ }^{13} \mathrm{C}$ NMR $\left(75 \mathrm{MHz}, \mathrm{CDCl}_{3}\right)$ : $\delta$ 178.0, 177.7, 176.9, 145.9, 145.5, 145.2, 144.9, $127.8,127.5,127.2,125.5,125.3,54.0,52.3,51.7$, $45.3,44.7,44.4,43.6,42.5,40.2,25.5,23.7,22.3$, $22.2,18.5,16.3,8.3 \mathrm{ppm} .{ }^{29} \mathrm{Si}$ NMR $(59.4 \mathrm{MHz}$, $\left.\mathrm{CDCl}_{3}\right): \delta-67.5,-67.8 \mathrm{ppm}$.

\section{Results and Discussion}

3.1. Triblock Terpolymer Synthesis by Living Anionic Polymerization

The desired PS- $b$-PMAPOSS- $b$-PMMA triblock terpolymers were prepared by living anionic polymerization in the presence of sce-BuLi as initiator, excess of $\mathrm{LiCl}$ and DPE at $-78{ }^{\circ} \mathrm{C}$. The first block consisted of PS followed by PMAPOSS as the second block and PMMA as the third block (Figure 1).

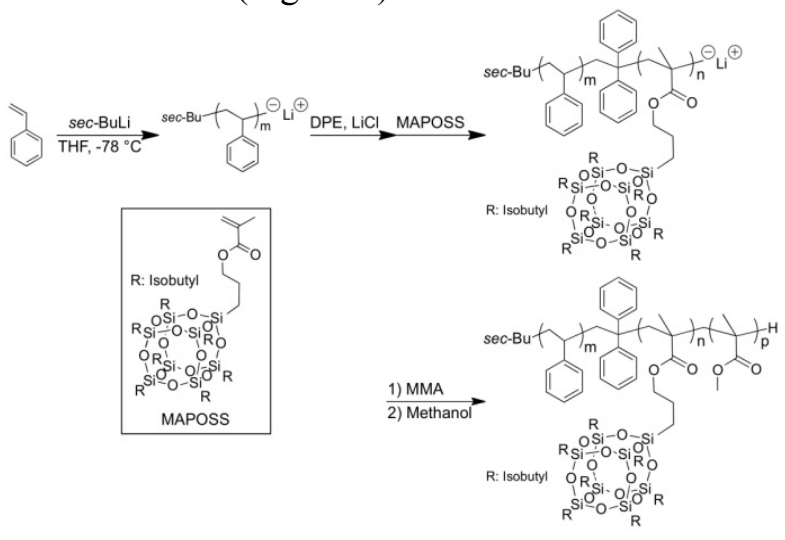

Figure 1. Synthetic scheme of PS- $b$-PMAPOSS- $b$-PMMA triblock terpolymer.

The polymerization results are listed in the table 1. The chemical structure of the triblock terpolymers were characterized by ${ }^{1} \mathrm{H},{ }^{13} \mathrm{C}$, and ${ }^{29} \mathrm{Si}$ NMR and IR spectra. The molecular weight 
was measured by SEC. Figure 2 shows ${ }^{1} \mathrm{H}$ NMR spectrum of PS- $b$-PMAPOSS- $b$-PMMA. The ${ }^{1} \mathrm{H}$ NMR spectrum of triblock terpolymer clearly show successful incorporation of each block, indicated by the appearance of methylene $\left(-\mathrm{OSiCH}_{2}\right)$ protons corresponding to PMAPOSS at $0.60 \mathrm{ppm}$, PS aromatic protons at 7.06-6.36 ppm and methyl groups protons corresponding to PMMA at $0.84 \mathrm{ppm}$. Also SEC chromatograms of the polymers show that molecular weights of the polymers increased in a controlled manner reflected by the shift higher molecular weight region (Figure 3). And the SEC analysis showed the molecular weight distribution is narrow (below 1.06). Hence, the composition of PS- $b$ PMAPOSS- $b$-PMMA was ascertained by using the integration ratio's from ${ }^{1} \mathrm{H}$ NMR spectrum of aromatic protons for PS, methylene protons for PMAPOSS and comparing with the integration of methoxy protons from PMMA along with the $M_{\mathrm{n}}$ values successfully obtained. The volume fraction of each block was estimated from the density of PS $\left(1.05 \mathrm{~g} \mathrm{~cm}^{-3}\right)$, that of PMAPOSS $\left(1.14 \mathrm{~g} \mathrm{~cm}^{-3}\right)$, and PMMA $\left(1.15 \mathrm{~g} \mathrm{~cm}^{-3}\right)$ in combination with the ${ }^{1} \mathrm{H}$ NMR data.

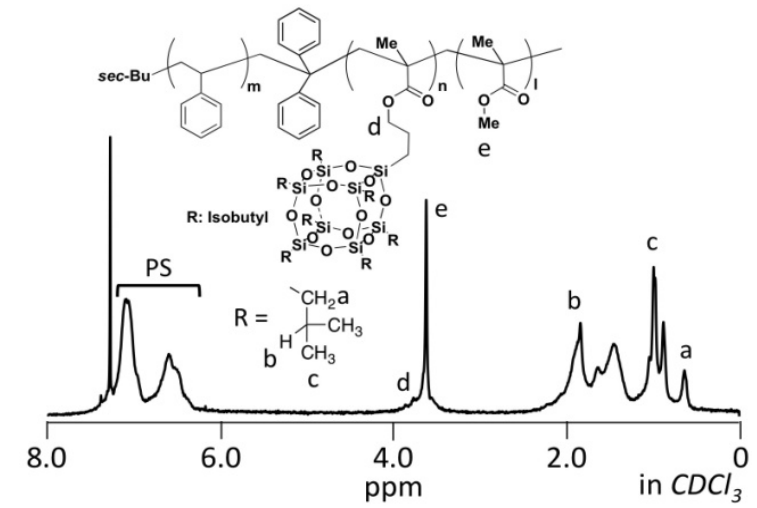

Figure 2. ${ }^{1} \mathrm{H} \quad$ NMR spectrum of PS- $b$-PMAPOSS- $b$-PMMA triblock terpolymer in $\mathrm{CDCl}_{3}$.

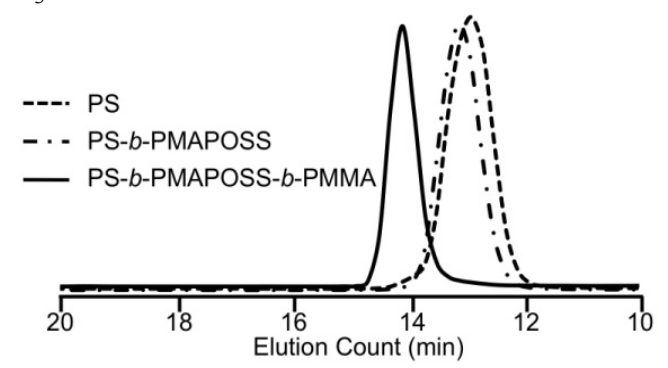

Figure 3. SEC curves of PS- $b$-PMAPOSS- $b$-PMMA (solid line), PS- $b$-PMAPOSS (meshed line), and PS (dashed line).

\subsection{Thermal Properties of Triblock Terpolymer} The thermal properties of
PS- $b$-PMAPOSS- $b$-PMMAs were investigated using TGA and DSC. We first confirmed that no significant weight loss was observed below $300{ }^{\circ} \mathrm{C}$ by TGA analysis. To eliminate the effect of thermal histories of the samples, the samples were heated to $200{ }^{\circ} \mathrm{C}$ and held for $10 \mathrm{~min}$ at this temperature before cooling to r.t at a rate of $10{ }^{\circ} \mathrm{C}$ $\mathrm{min}^{-1}$. DSC data reported was obtained from the second run. Two transitions were observed at $87^{\circ} \mathrm{C}$ and $99^{\circ} \mathrm{C}$ in the heating cycle, which are corresponding to the $T_{\mathrm{g}}$ of the PMMA and PS, respectively.

Table 1. Characteristics of Triblock Terpolymers ${ }^{a}$ Measured by SEC, relative to PS linear standards in

\begin{tabular}{ccccc}
\hline Run & $M_{\mathrm{n}}{ }^{\mathrm{a}}$ & $M_{\mathrm{w}} / M_{\mathrm{n}}{ }^{\mathrm{a}}$ & $\mathrm{wt}^{\mathrm{b}}{ }^{\mathrm{b}}$ & vol $^{\mathrm{c}}$ \\
\hline 1 & 47100 & 1.03 & $30 / 43 / 27$ & $32 / 42 / 26$ \\
2 & 58800 & 1.04 & $28 / 12 / 60$ & $30 / 12 / 58$ \\
3 & 45000 & 1.04 & $34 / 17 / 49$ & $36 / 17 / 47$ \\
4 & 82000 & 1.05 & $32 / 23 / 45$ & $34 / 22 / 44$ \\
5 & 56000 & 1.06 & $31 / 28 / 42$ & $33 / 27 / 40$ \\
\hline
\end{tabular}

THF. ${ }^{\mathrm{b}}$ The numbers refer to the final composition determined by SEC calibrated against PS linear standard and integrations from ${ }^{1} \mathrm{H}$ NMR spectrum.

\subsection{Bulk Morphological Characterization}

The morphologies of the PS- $b$-PMAPOSS- $b$-PMMAs in their bulk state were studied using SAXS, and TEM. The samples were prepared by slow evaporation from chloroform at room temperature, then thermal annealed at $180{ }^{\circ} \mathrm{C}$ for $24 \mathrm{~h}$ in a vacuum oven.

At first, we investigated the micro phase separation structure of the PS- $b$-PMAPOSS- $b$-PMMA 1, which has the composition creating the three-domain lamella in the reported triblock terpolymer such as PS- $b$-poly(isoprene)- $b$-PMMA and PI- $b$ PS- $b$-poly(ethylene oxide) $[10,11]$. The SAXS profile displayed five scattering peaks, which ratio was $1: 3^{1 / 2}: 2: 3: 5$. Figure $4 \mathrm{~b}$ shows a TEM image of PS- $b$-PMAPOSS- $b$-PMMA 1 sample after staining with $\mathrm{RuO}_{4}$. In the TEM image, there are bright dots of PMMA with in PMAPOSS phase, which seem to cause the undulation of the lamellae. The SAXS pattern of the solution clearly exhibits the peaks characteristic of a lamellar structure, while this sample the [110] reflection observed. Considering the volume fractions of the blocks, we assume an undulatedlamellar-morphology with PMMA-core surrounded by a PMAPOSS-layer within a 
PS-layer. And, the PMMA minority phase forms hexagonally arranged dots within the PMAPOSS sheets of a lamellar phase.
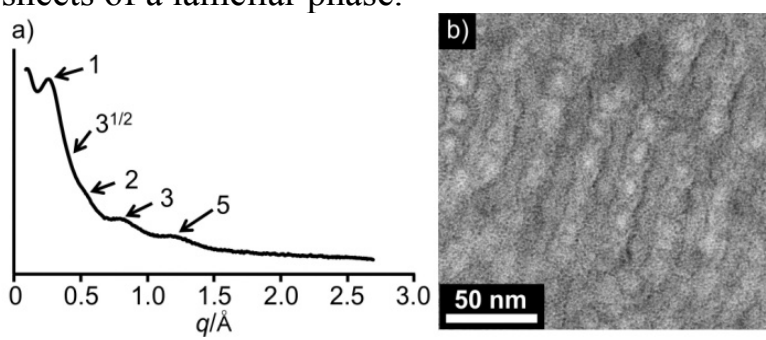

Figure 4. SAXS and TEM results of PS- $b$-PMAPOSS- $b$-PMMA 1, a)SAXS profile, and b)TEM image.

While, reduction of the PMAPOSS volume fraction, PS- $b$-PMAPOSS- $b$-PMMA 2-4, the SAXS diffraction profiles exhibited reflections based on lamellar structure. In addition, the TEM images of PS- $b$-PMAPOSS- $b$-PMAHFC 2-4 showed two-domain lamellar nanostructures, which is strongly supported by the SAXS results. In TEM measurement, samples were imaged without staining as the contrast between the POSS-containing block (dark regions), the PS block and the PMMA block (bright regions) was sufficient for achieving high-quality images (Figure 5a). Then, in order to investigate whether the obtained nanostructure is thermodynamically stable structure, we prepared the PS- $b$-PMAPOSS- $b$-PMMA 3 sample extending thermal annealing time for $48 \mathrm{~h}$. As the result of TEM measurement, no change was seen, indicated that the annealing condition could give forming a thermodynamically stable nanostructure (Figure $5 b)$. Moreover, no change was observed in the domain size and contrast image before and after staining with $\mathrm{RuO}_{4}$ (Figure 5c). Thus, the lamellar morphology of an AB-like block copolymer, in which PS and PMAPOSS form the mixed phase between two PMMA layers.

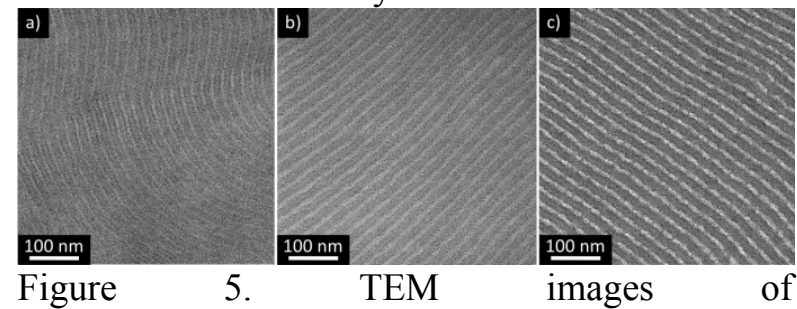

PS- $b$-PMAPOSS- $b$-PMMA 3, a)thermal annealed at $180{ }^{\circ} \mathrm{C}$ for $24 \mathrm{~h}, \mathrm{~b}$ )thermal annealed at $180^{\circ} \mathrm{C}$ for $48 \mathrm{~h}$, and c)stained with $\mathrm{RuO}_{4}$ (bright phase PMMA).

Further increasing of the PMAPOSS volume fraction leads to the formation of a three-domain lamellar nanostructure. This can be seen in Figure
6, which shows a TEM micrograph. In this case, all three block components can be distinguished: PMMA appears as white phase, the grey phase assigned to PS, and the very narrow dark phase can be assigned to PMAPOSS. The lamellar $d$-spacing was found to be $34 \mathrm{~nm}$, which is in good agreement with values obtained from the TEM image. And, the width of remarkable narrowly dark domain is $4 \mathrm{~nm}$. The lamellar structure is the layered structure whose repeating unit is $-\mathrm{ABCB}^{-}$, A, B and $\mathrm{C}$ is PS, PMAPOSS, and PMMA, respectively (Figure 6b). The ultra narrow PMAPOSS layer, which is not obtained in the PMAPOSS containing diblock copolymer system, was observed in a designed triblock terpolymer, which is the result of the highly repulsive PMAPOSS middle segment.

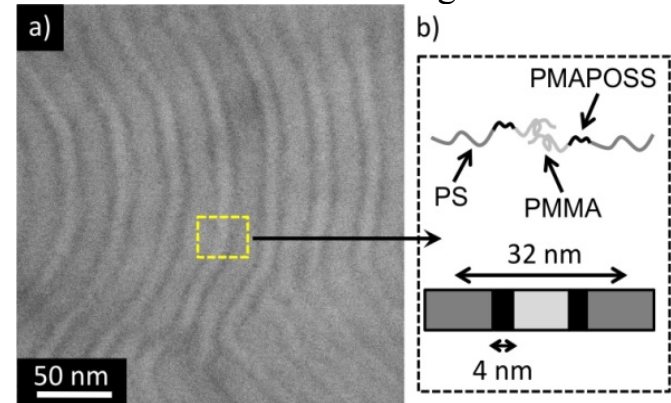

Figure 6. TEM image and schematic illustration; a)TEM image of PS- $b$-PMAPOSS- $b$-PMMA 5, b)schematic illustration of three-domain layer.

\section{Conclusion}

In conclusion, a new series of POSS-containing triblock terpolymers, PS- $b$-PMAPOSS- $b$-PMMAs, was prepared using living anionic polymerization. The use of this method enables control of the molecular weight and the achievement of narrow molecular weight distributions. We also successfully obtained the ultra narrow PMAPOSS layer $(4 \mathrm{~nm})$ in the bulk of the PS- $b$-PMAPOSS- $b$-PMMA lamellar morphology. This morphology is expected to be a promising line patterns in the block copolymer lithography.

\section{Acknowledgements}

We thank Ryohei Kikuchi, National University Corporation Tokyo Institute of Technology Center for Ascended Materials Analysis, for the TEM. .

\section{References}

1. C. J. Hawker, T. P. Russell, MRS Bulletin 3 (2005), 952.

2. M. Lazzari, M. A. Lopez-Quintela, $A d v$. Mater. 15 (2003), 1583.

3. J. Y. Cheng, C. A. Ross, H. I. Smith, E. L. 
Thomas, Adv. Mater. 18 (2006), 2505.

4. S. O. Kim, H. H. Solak, M. P. Stoykovich, N. J. Ferrier, J. J. DePablo and P. F. Nealey. Nature 424 (2003) 411.

5. R. A. Segalman, H. Yokoyama, E. J. Kramer, Adv. Mater. 13 (2001), 1152.

6. T. Hirai, M. Leolukman, S. Jin, R. Goseki, Y. Ishida, M. Kakimoto, T. Hayakawa, M. Ree, P. Gopalan, Macromolecules 42 (2009), 8853.

7. T. Hirai, M, Leolukman, C. C. Liu, E. Han, Y. J. Kim, Y. Ishida, T. Hayakawa, M. Kakimoto, P. F. Nealey, P. Gopalan,
Adv. Mater. 21 (2009), 4337.

8. R. Goseki, T. Hirai, Y. Ishida, M. Kakimoto, T. Hayakawa, Polymer Journal 44 (2013), 658.

9. Y. Tada, H. Yoshida, Y. Ishida, T. Hirai, J. K. Bosworth, E. Dobisz, R. Ruiz, M. Takenaka, T. Hayakawa, H. Hasegawa, Macromolecules 45 (2012), 292.

10. Y. Mogi, M. Nomura, H. Kotsuji, K. Ohnishi, Y. Matsushita, I. Noda, Macromolecules 27 (1994), 6755.

11. J. Chatterjee, S. Jain, F. S. Bates, Macromolecules 40 (2007), 2882. 